\author{
Military Technical College \\ Kobry El-Kobbah, \\ Cairo, Egypt.
} $13^{\text {th }}$ International Conference
on Applied Mechanics and
Mechanical Engineering.

\title{
NON-LINEAR FORCED VIBRATION OF A TAPERED CANTILEVER BEAM
}

\author{
AL-QAISIA* A.A. and ABDEL-JABER ${ }^{\star *}$ M.S.
}

\begin{abstract}
This paper presents a study on the nonlinear frequency response of a tapered cantilever beam. The mathematical model is developed based on the large deformation theory, nonlinear curvature and with the constraint of inextensible beam. The Lagrangian dynamics with the assumed mode method is utilized in deriving the nonlinear, uni-modal temporal equation of motion. The non-linear equation of motion is solved analytically using the harmonic balance method (HB). Results were obtained for tapered beams with different values of taper ratio. The effect of taper ratio and excitation level on the qualitative behaviour of the forced response of the beam were studied and presented the first three modes of vibration.
\end{abstract}

\section{KEY WORDS}

Tapered Beams, Non-Linear, Forced Vibration.

* Department of Mechanical Engineering, Faculty of Engineering and Technology, University, of Jordan, Amman, Jordan.

** Department of Civil Engineering, Faculty of Engineering and Technology, University of Jordan, Amman, Jordan 


\section{INTRODUCTION}

It is known that a lot of engineering structures can be modelled as tapered beams, such as piles, fixed-type platforms, tower structures, high buildings and robot arms.

In general, due to various excitation loads "wind and waves", high aspect ratio and flexibility such structures might have large deformations and deflections. The prediction of the dynamic behaviour is extremely important during the design process and operations.

The linear vibration theory predicts the natural frequencies to be independent of the amplitude. But in many cases, the deflection in structures may reach large values and consequently, using the linear vibration assumption is not valid. In order to take into consideration the nonlinearities arised due to large deformations, the nonlinear vibration theory must be used to predict with high accuracy the dynamic behaviour like; natural frequencies and dynamic responses.

In literature much research was devoted to calculate the linear natural frequencies and mode shapes [1-6] with different end conditions and with attached inertia elements at the free end of the beam. In [7], a simple formulation for the large amplitude free vibrations of tapered beams was presented. The method is based on an iterative numerical scheme to obtain results for tapered beams with rectangular and circular cross sections.

The objective of the present work is to study the nonlinear planar large amplitude forced vibration of a cantilever tapered beam. The mathematical model is derived using the Lagrange method and the resulting continuous equation is discretized using the assumed mode method. The inextensibility condition is used to relate the axial shortening due to transverse deflection in the formulation of the kinetic energy of the beam and the nonlinear curvature is used in the potential energy expression [10]. The steady state frequency response is obtained using the harmonic balance method [9].

\section{MATHEMATICAL MODEL}

A schematic of the tapered beam under study is shown in Figure 1. The physical properties, modulus of elasticity $E$ and density $\rho$, of the beam are constants. While the beam thickness and width are varied linearly along the beam axis. The beam is clamped at one end and free at the other, the cross sectional area and moment of inertia at the large (Clamped end) are $A_{b 1}$ and $I_{b 1}$, respectively.

The thickness of the beam is assumed to be small compared to the length of the beam, so that the effects of rotary inertia and shear deformation can be ignored. The beam transverse vibration can be considered to be purely planar and the amplitude of vibration may reach large values.

Using the deformed beam, see Figure 2, the potential energy of the beam can be written as 


$$
V=\frac{E l}{2} \int_{0}^{1} I(\zeta) R^{2} d \zeta
$$

where $\zeta=s / l, I(\zeta)$ is the variable second moment of area and $R$ is the curvature of the beam neutral axis and can expressed as $[8,10]$

$$
R=\lambda \phi^{\prime}
$$

where $\lambda=1 / l$, the prime is the derivative with respect to the dimensionless length, $\zeta$, and $\varphi$ is the slope of the deformed beam (see Figure 2). The exact curvature can be expressed in terms of the transverse deflection, $v$, by using $\cos \phi=\sqrt{1-\sin ^{2} \phi}$, where $\sin \phi=\mathrm{d} v / \mathrm{d} s=\lambda v^{\prime}$ (as in Figure 2). Differentiating with respect to $\zeta$, using the above trigonometric identities and expanding the resulted term and retaining the terms up to the fourth order, the nonlinear curvature $R$ can be written as

$$
R^{2}=\lambda^{4}\left(v^{\prime \prime 2}+\lambda^{2} v^{\prime 2} v^{\prime 2}\right)
$$

The kinetic energy $T$ of the deformed beam during motion can be written as

$$
T=\frac{1}{2} \rho l \int_{0}^{1} A(\zeta)\left[\dot{u}^{2}+\dot{v}^{2}\right] \mathrm{d} \zeta
$$

where $u$ is the axial shortening due to bending deformation as can be seen in Figure 2 . The inextensibility condition dictates that a total axial shortening $u$ is given by [10]

$$
\lambda u=\zeta-\int_{0}^{\zeta} \cos \phi d \zeta=\zeta-\int_{0}^{\zeta} \sqrt{1-\left(\lambda v^{\prime}\right)^{2}} d \zeta
$$

Expanding the radical term in a power series, assuming that $\left(\lambda v^{\prime}\right)^{2}<<1$, the axial shortening can be represented as

$$
u=\frac{1}{2} \int_{0}^{\zeta}\left(\lambda v^{\prime 2}+\frac{\lambda^{3}}{4} v^{\prime 4}\right) \mathrm{d} \zeta
$$

Differentiating Eq.(6) with respect to time yields

$$
\dot{u}=\frac{1}{2} \frac{d}{d t} \int_{0}^{\zeta}\left(\lambda v^{\prime 2}\right) \mathrm{d} \zeta
$$

Since the Lagrangian ( $L=T-V$ ) of the beam is continuous and does not admit a closed form solution, the interest here is in the case where the beam motion is governed by single active mode. The Lagrangian of the system $L$ can be discretized by using the assumed mode method and substituting 


$$
v(\zeta, t)=\phi_{i}(\zeta) q(t)
$$

where $\phi_{i}(\zeta)$ is $i$-th normalized mode shape of the beam and $q(t)$ is an unknown time modulation of the assumed mode. The $i$-th mode shape $\phi_{i}(\zeta)$ for a double tapered beam is given by [6]:

$$
\phi_{i}(\zeta)=\zeta^{-1}\left[C_{1} J_{2}(Z)+C_{2} Y_{2}(Z)+C_{3} I_{2}(Z)+C_{4} K_{2}(Z)\right]
$$

where $A(\zeta)=A_{b l} \zeta^{2}$ and $I(\zeta)=I_{b l} \zeta^{4}$, and for wedge-type beams (single taper)

$$
\phi_{i}(\zeta)=\zeta^{-1 / 2}\left[C_{1} J_{1}(Z)+C_{2} Y_{1}(Z)+C_{3} I_{1}(Z)+C_{4} K_{1}(Z)\right]
$$

And $A(\zeta)=A_{b l} \zeta$ and $I(\zeta)=I_{b l} \zeta^{3}$

For both cases $Z=2 \beta \zeta^{1 / 2}, \beta^{4}=\frac{\rho A_{b l} L_{l}^{4} \omega_{l}^{2}}{E I_{b 1}}, \omega_{l}$ is the linear frequency of vibration, $J$ and $Y$ are Bessel functions of the first and second kind, respectively, and $I$ and $K$ are modified Bessel functions of the first and second kind, respectively. $C_{1}, C_{2}, C_{3}$ and $C_{4}$, are arbitrary constants to be determined form the four boundary conditions imposed to both ends of the beam; zero bending moment and zero shear force at the free end and zero deflection and zero slope at the clamped end.

$$
\begin{gathered}
E I(\zeta) \phi_{i}^{\prime \prime}\left(\zeta_{0}\right)=0 \\
\frac{\mathrm{d}}{\mathrm{d} \zeta}\left(E I(\zeta) \phi_{i}^{\prime \prime}\left(\zeta_{0}\right)\right)=0 \\
\phi_{i}^{\prime}(1)=0 \\
\phi_{i}(1)=0
\end{gathered}
$$

Using Eqs. (7-10), the Lagrangian expression $L$ of the tapered beam, for the $i$-th mode of vibration, can be written as;

$$
L=\rho l^{3}\left(\beta_{1} \dot{q}^{2}+\beta_{2} q^{2} \dot{q}^{2}-\beta^{2} \beta_{3} q^{2}-\beta^{2} \beta_{4} q^{4}\right)
$$

where

$$
\begin{gathered}
\beta_{1}=\int_{0}^{1} A_{1}^{*} \phi^{2} \mathrm{~d} \zeta \\
\beta_{2}=\int_{0}^{1} A_{1}^{*}\left\{\int_{0}^{\zeta} \phi^{\prime 2} \mathrm{~d} \chi\right\}^{2} \mathrm{~d} \zeta
\end{gathered}
$$




$$
\begin{gathered}
\beta_{3}=\int_{0}^{1} I_{1}^{*} \phi^{\prime \prime 2} d \zeta \\
\beta_{4}=\int_{0}^{1} I_{1}^{*} \phi^{\prime 2} \phi^{\prime \prime 2} \mathrm{~d} \zeta
\end{gathered}
$$

For the double tapered beam; $A_{1}^{*}=A_{b l} \zeta^{2}$ and $I_{1}^{*}=I_{b l} \zeta^{4}$ and for single tapered beam, the wedge beam $A_{1}^{*}=A_{b 1} \zeta$ and $I_{1}^{*}=I_{b 1} \zeta^{3}$.

Applying the Euler-Lagrangian equation to the system Lagrangian

$$
\frac{\mathrm{d}}{\mathrm{d} t}\left(\frac{\partial L}{\partial \dot{q}}\right)-\frac{\partial L}{\partial q}=0
$$

the following non-linear, non-dimensional uni-modal equation of motion is obtained:

$$
\beta_{1} \ddot{q}+\beta_{2}\left(q^{2} \ddot{q}+q \dot{q}^{2}\right)+\beta^{2}\left(\beta_{3} q+2 \beta_{4} q^{3}\right)=0
$$

Due to the fact that, some of the coefficients $\beta_{i}$, defined by Eqs. 13-16, may have large values, Eq. (18) for convenience is scaled to the form;

$$
\ddot{q}+q+\varepsilon_{1}\left(q^{2} \ddot{q}+q \dot{q}^{2}\right)+\varepsilon_{2} q^{3}=0
$$

A dot is used to denote a derivative with respect to the non-dimensional time. $t^{*}=\left(\beta^{2} \beta_{3} / \beta_{1}\right)^{1 / 2} t, \varepsilon_{1}=\frac{\beta_{2}}{\beta_{1}}$ and $\varepsilon_{2}=\frac{2 \beta_{4}}{\beta_{3}}$ are dimensionless coefficients.

Eq.(19) describes the non-linear non-dimensional planar flexural free vibration of the inextensible tapered beam. In this equation, the terms $\varepsilon_{1} \ddot{q} q^{2}$ and $\varepsilon_{1} q \dot{q}^{2}$ are inertia nonlinearities arised from using the inextensibility condition in the kinetic energy and they are of softening type. The non-linear term $\varepsilon_{2} q^{3}$ is due to the potential energy stored in bending and arises as a result of using non-linear curvature and it is of hardening static.

\section{METHOD OF SOLUTION}

To study the nonlinear forced vibration of the tapered beam system under consideration, an excitation term can be added to the right hand side of equation (19), such that

$$
\ddot{q}+q+\varepsilon_{1}\left(q^{2} \ddot{q}+q \dot{q}^{2}\right)+\varepsilon_{2} q^{3}=P \cos \left(\Omega t^{*}\right)
$$

where $P$ and $\Omega$, are the excitation level and frequency respectively. 
Approximate analytical solutions for the periodic steady state response, having the same period as the excitation, of the nonlinear oscillator described by equation (20) can be obtained using a single mode harmonic balance method (SHB). For the sake of simplicity, a new time $T=\Omega t^{*}$ is first introduced so that equation (20) becomes;

$\Omega^{2} \ddot{q}+q+\varepsilon_{1} \Omega^{2}\left(q^{2} \ddot{q}+q \dot{q}^{2}\right)+\varepsilon_{2} q^{3}=P \cos (T+\phi)$

where dots are $T$ derivative and the unknown phase $\phi$ has been added to the excitation so that one may obtain a fundamental harmonic response containing a single trigonometric term.

According to the SHB method, an approximate solution of equation (21), takes the form;

$$
q(T)=A \cos T
$$

where $A$ is the steady state response amplitude. Substituting equation (22) into equation (21), neglecting third harmonics, which arise, and equating coefficients of first harmonics, one obtains the following equations:

$$
\begin{gathered}
\left(\frac{3}{4} \varepsilon_{2}-\frac{\varepsilon_{1}}{2} \Omega^{2}\right) A^{3}+\left(1-\Omega^{2}\right) A=P \cos \phi \\
-\Omega \delta A=P \sin \phi
\end{gathered}
$$

The steady state frequency response is obtained by squaring and adding equations (23) and (24) and solving for $\Omega^{2}$ as a function of $A$; this yields

$$
\Omega^{2}=R_{1} \pm \sqrt{R_{1}^{2}-R_{2}}
$$

where

$$
\begin{gathered}
R_{1}=-\left(\delta^{2}-\frac{3}{2} \varepsilon_{2} A^{2}-\frac{3}{4} \varepsilon_{1} \varepsilon_{2} A^{4}-2-\varepsilon_{1} A^{2}\right) /\left(2+2 \varepsilon_{1} A^{2}+\frac{\varepsilon_{1}^{2} A^{4}}{2}\right) \\
R_{2}=\left(\frac{9}{16} \varepsilon_{2}^{2} A^{4}+\frac{3}{2} \varepsilon_{2} A^{2}+1-\frac{P^{2}}{A^{2}}\right) /\left(1+\varepsilon_{1} A^{2}+\frac{\varepsilon_{1}^{2} A^{4}}{4}\right)
\end{gathered}
$$

Equation (25), yields two real solutions for $\Omega$ provided that the radical term is real and less than $R_{1}$; a single real solution is obtained when the radical term is zero or greater the $R_{1}$, and no real solution exists when $R_{1}^{2}-R_{2}<0$. 


\section{RESULTS AND DISCUSSION}

The coefficients of the terms $\beta_{i}$ given in Eq. (18) are calculated by integrating numerically the coefficients given in Eqns. (13-16).

The frequency response of the non-linear oscillators governed by equation (21) was calculated, for given values of taper ratios $\alpha$ and excitation levels $P$ using the single term harmonic balance method (SHB). Examples of the results of these calculations are presented Figures (3-6).

In Figure 3, the steady state frequency responses were obtained for different values of taper ratio $\alpha=b_{0} / b_{1}$ and for excitation $P=5$. It is clear that, increasing the taper ratio $\alpha$ will change the qualitative behaviour of the response from hardening to a softening type, and this due to the fact that steady state frequency response exhibits a softening behaviour when, roughly, $\varepsilon_{1} / \varepsilon_{2}>1.6$, hardening behaviour when $\varepsilon_{1} / \varepsilon_{2}<1.6$, and resembles linear behaviour when $\varepsilon_{1} \cong 1.6 \varepsilon_{2}$ [9].

In Figures (4-6), the frequency responses were obtained for the first three modes of vibration and for different values of taper ratio $\alpha$. Results have shown that, for the first and second modes, the behaviour is changed from hardening to softening, depending on the value of the taper ratio. While the third and higher modes are of softening type regardless the value of the taper ratio.

\section{CONLUSIONS}

A mathematical model of a tapered beam cantilever beam is derived. The axial shortening due to transverse deflection and the nonlinear curvature are used in the formulation of the kinetic and potential energy, respectively. The assumed mode method is used to discretize the continuous Lagrangian of the system and the resulted uni-modal nonlinear differential equation of motion is solved using the Harmonic Balance method (HB).

Results have shown that, for the first and second modes the behavior is changed from hardening to softening type when the taper ratio $\alpha$ is increased, while the third mode is of a softening type regardless the value of the taper ratio $\alpha$.

\section{ACKNOWLEDGMENT}

The authors acknowledge the support of the University of Jordan.

\section{REFERENCES}

[1] Auciello, N. M. and Nole, G. "Vibrations of a Cantilever Tapered Beam with varying section properties and Carrying a Mass at the Free End", Journal of Sound and Vibration, 1998, 214, pp. 105-119.

[2] Nagaya, K. and Hai, Y. "Seismic Response of Underwater members of 
Variable Cross Section", Journal of Sound and Vibration, 1985, 119, pp. 119138.

[3] Laura, P. A.and Gutierrez, R. H., "Vibrations of an Elastically restrained Cantilever Beam of Varying Cross Sections with Tip Mass of Finite Length", Journal of Sound and Vibration, 1986, 108, , pp. 123-131.

[4] Shong, J. W. and Chen, C.T., "An Exact Solution for the Natural Frequency and Modes Shapes of an Immersed Elastically Wedge Beam Carrying an Eccentric Tip Mass with Mass Moment of Inertia", Journal of Sound and Vibration, 2005, 286, pp. 549-568.

[5] Chen, D. W. and Wu, J. S., "The Exact Solutions for the Natural Frequency and Modes Shapes of Non-Uniform Beams with Multiple Spring-Mass Systems", Journal of Sound and Vibration, 2002, 255, pp. 299-322.

[6] Goorman, D. J., "Free Vibrations of Beams and Shafts", John-Wiley \& Sons, 1975, pp. 365.

[7] Rao, B. N. and Rao, G. V., "Large Amplitude Vibrations of a Tapered Cantilever Beam", Journal of Sound and Vibration, 1988, 127, pp. 173-178.

[8] Al-Qaisa, A. A. , Shatnawi, A., Abdel-Jaber, M. S., Abdel-Jaber M. and Sadder, S., "Non-Linear Natural Frequencies of a Tapered Cantilever Beam",. Proceedings of the Sixth International Conference on Steel and Aluminum Structures (ICSAS'07), Oxford, UK, July 24-27, 2007, pp. 266-273.

[9] Al-Qalisia, A. A. and Hamdan, M. N., "On the Steady State Response of Oscirlators with Static and Inertia Non-Linearities", Journal of Sound and Vibation, 1999, 223, pp. 49-71.

[10] Al-Qaisia, A. A. and Hamdan, M. N., "Bifurcation and Chaos of an Immersed Cantilever Beam in a Fluid and Carrying an Intermediate Mass", Journal of Sound and Vibration, 2002, 253, pp. 859-888. 


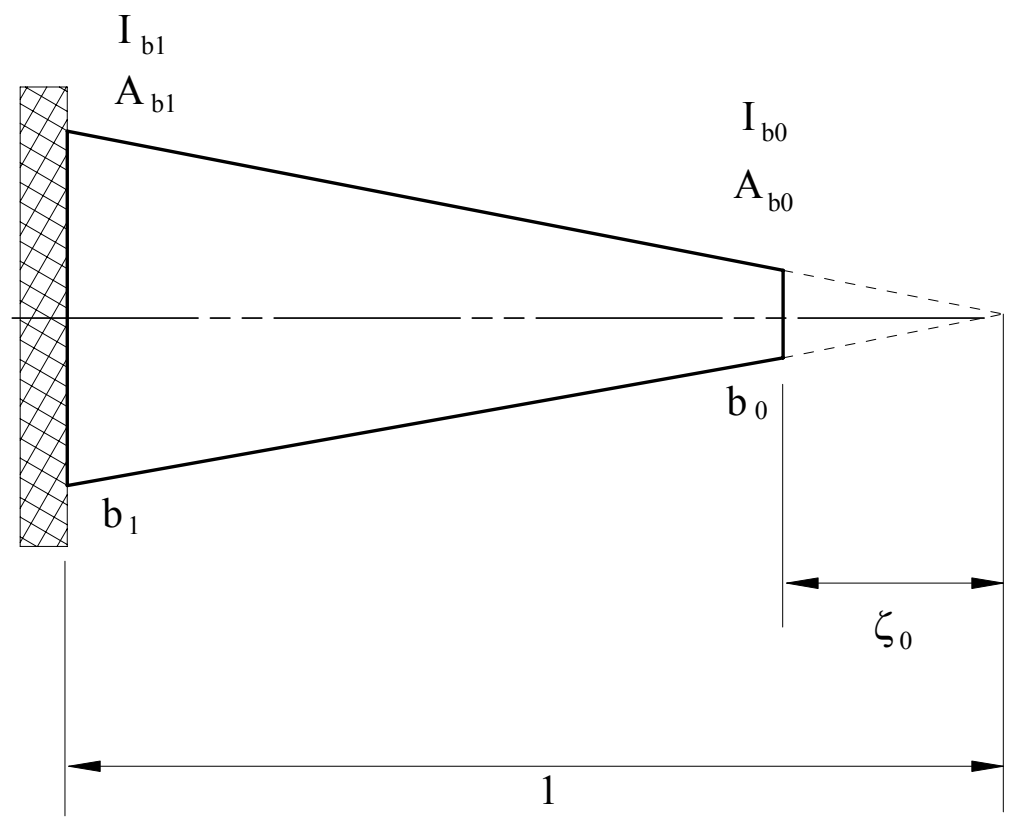

Figure (1), A schematic drawing of the tapered cantilever beam

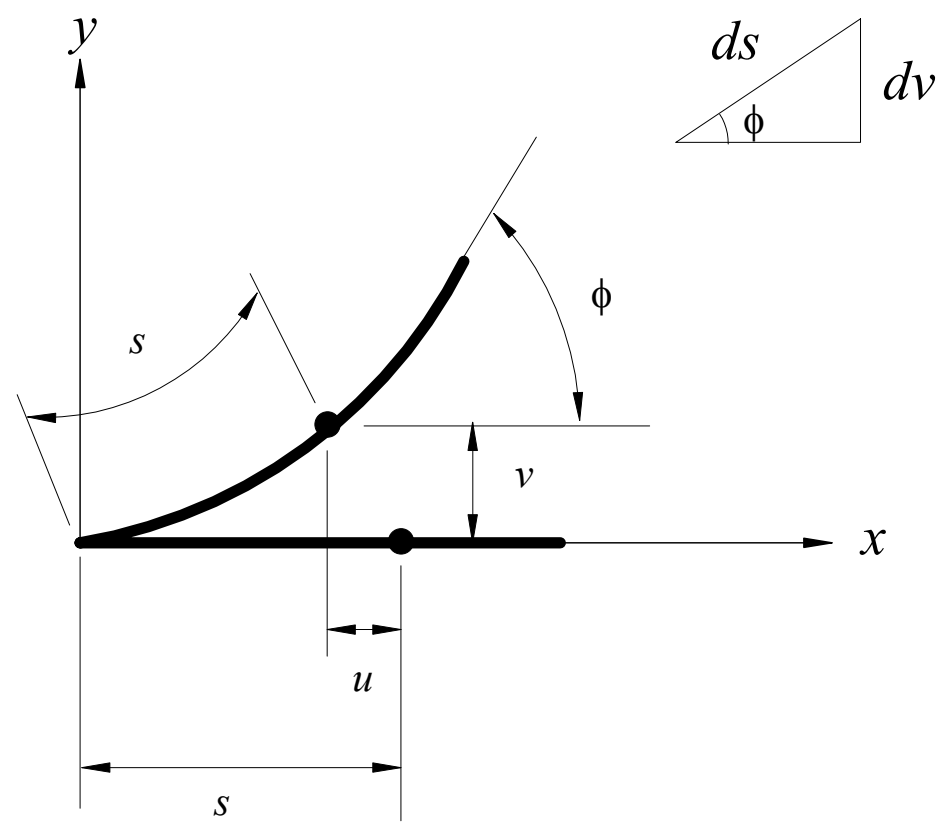

Figure 2. The deformed inextensible beam 


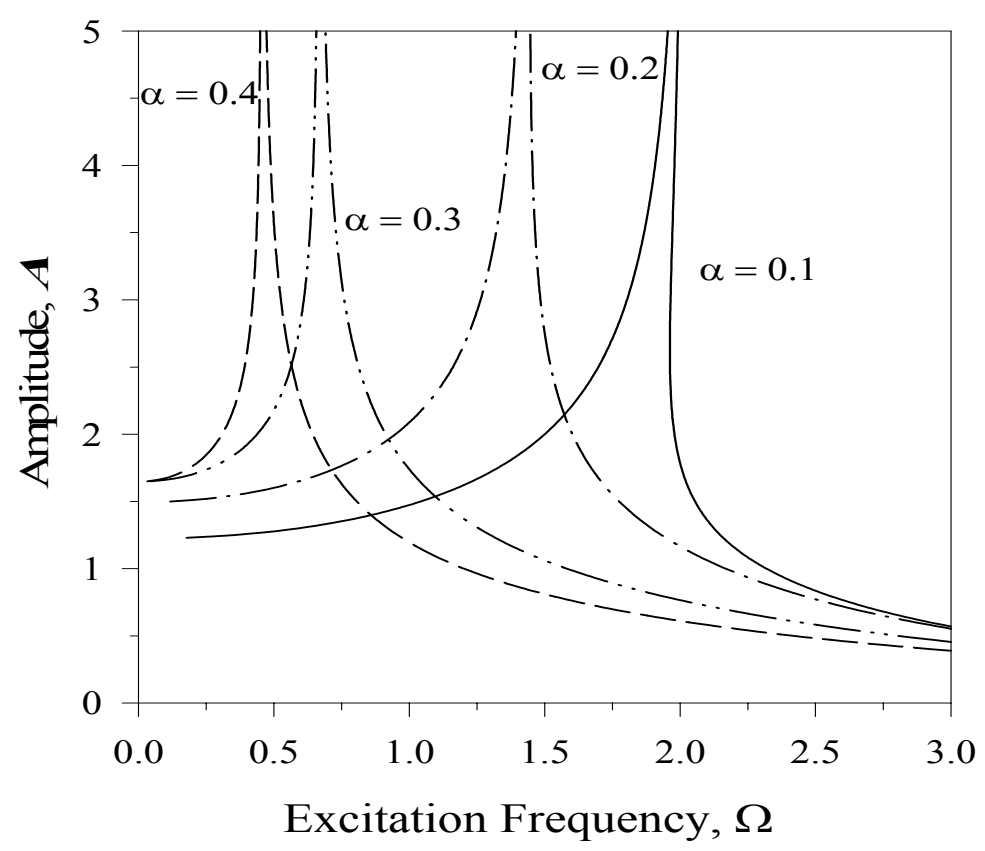

Figure (3), Frequency response of the first mode for different values of taper ratio $\alpha$ and for $P=5$.

$$
\alpha=0.1
$$

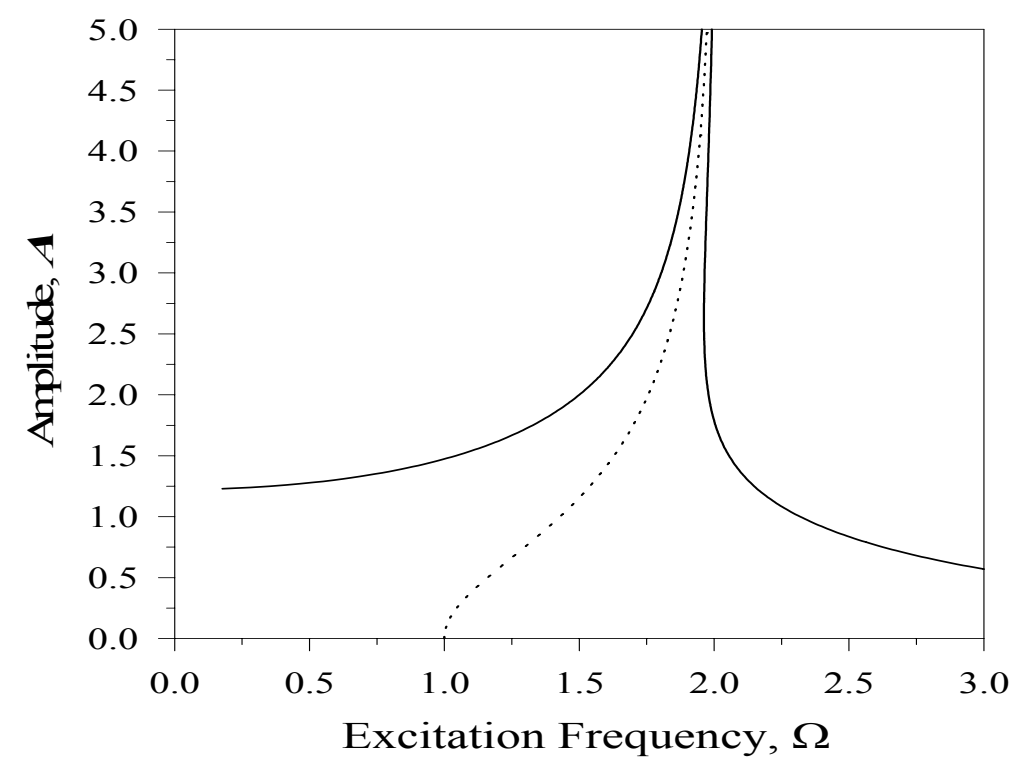

Figure (4), Frequency response of the first mode for $\alpha=0.1$ and for $P=5$. 


$$
\alpha=0.3
$$

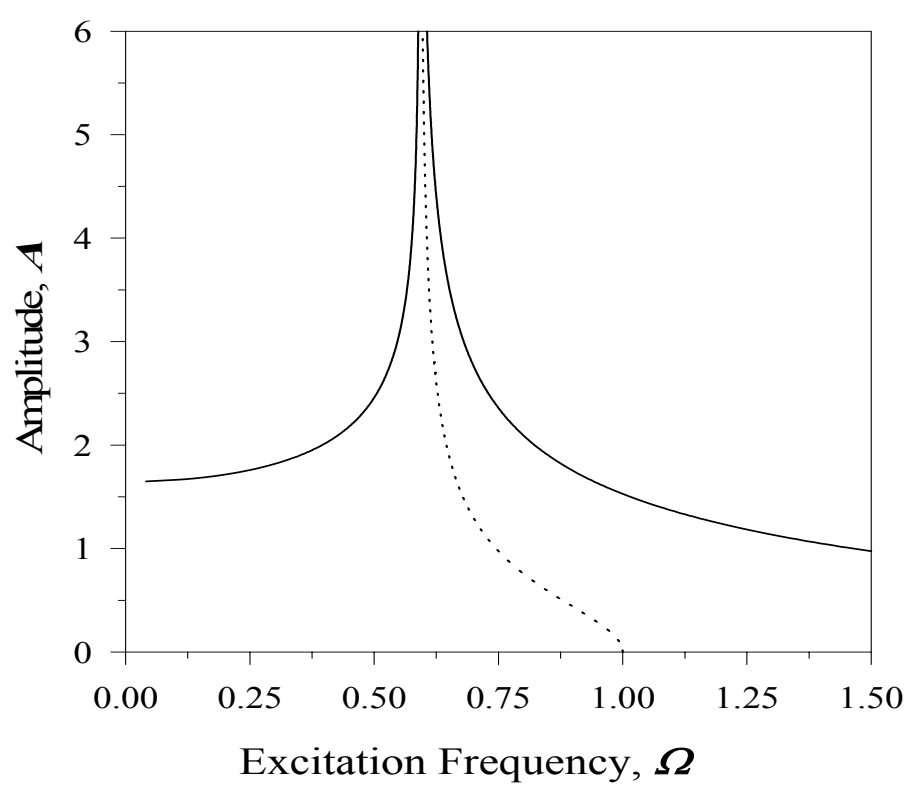

Figure (5), Frequency response of the second mode for $\alpha=0.3$ and for $P=5$.

$$
\alpha=0.7
$$

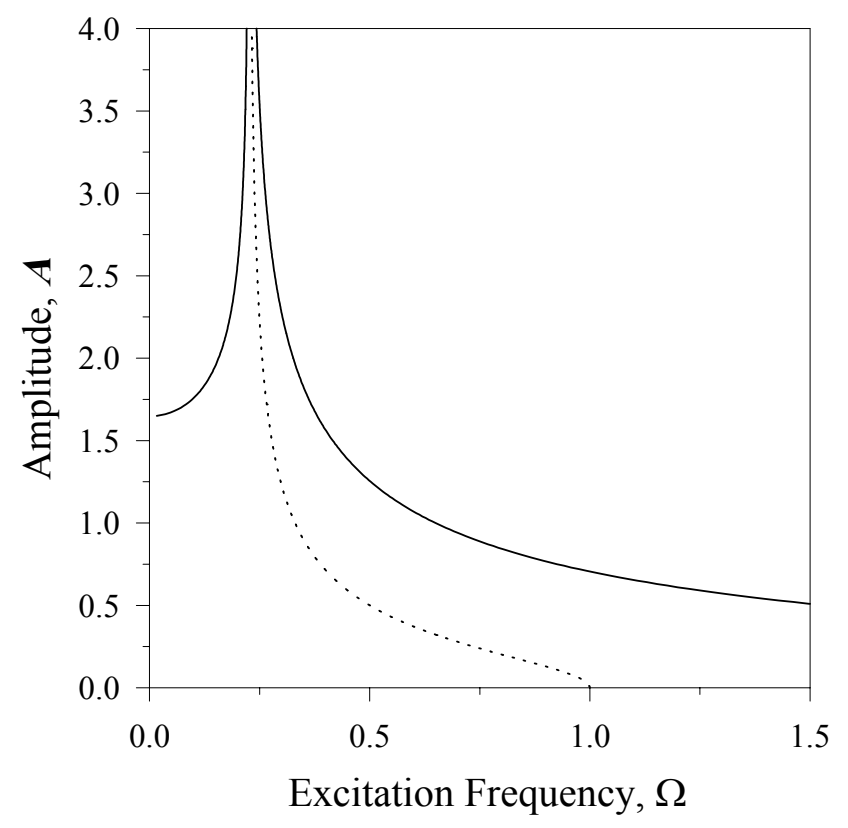

Figure (6), Frequency response of the third mode for $\alpha=0.7$ and for $P=5$. 